\title{
ON INVARIANT SUBSPACES OF NORMAL OPERATORS ${ }^{1}$
}

\author{
JOHN WERMER
}

1. Introduction. In this paper we are concerned with a special case of the following general problem: Let $B$ be a topological linear space and let $T$ be a bounded linear operator on $B$ which possesses a set of eigen-vectors fundamental in $B$. Under what conditions will every closed subspace $C$ of $B$ which is invariant under $T$ (in the sense that if $x \in C$ also $T x \in C$ ) contain a set of eigen-vectors spanning it? If a given operator has this property, we shall say that "spectral synthesis" holds for the operator.

It is easy to see that if $B$ is finite-dimensional, spectral synthesis holds for every linear operator on $B$ whose eigen-vectors span $B$. For let $\lambda_{1}, \lambda_{2}, \cdots, \lambda_{m}$ be the distinct eigen-values of $T$. Let $N_{i}$ be the subspace of eigen-vectors belonging to $\lambda_{i}$. Then $B$ decomposes into the complementary subspaces $N_{1}, N_{2}, \cdots, N_{m}$. Let $C$ be any invariant subspace. Given $x$ in $C$ we write $x=\sum_{1}^{m} \chi_{i}$, where $\chi_{i} \in N_{i}$. Let now $L$ be a linear functional on $B$ which vanishes on $C$. Then $L\left(T^{k} x\right)=\sum_{1}^{m} \lambda_{i}^{k} L\left(\chi_{i}\right)=0$ for all $k \geqq 0$. Since the Vandermonde determinant does not equal 0 , we must have $L\left(\chi_{1}\right)=\cdots=L\left(\chi_{m}\right)$ $=0$. Hence each $\chi_{i} \in C$. Thus every vector in $C$ is a sum of eigenvectors belonging to $C$, as asserted.

In this paper we shall study the problem of spectral synthesis for bounded normal operators on Hilbert space. We shall also study a certain generalization of spectral synthesis which we call property (P).

2. TheOREM 1. Let $T$ be a bounded normal operator on Hilbert space $H$. Then the following conditions are each equivalent to spectral synthesis:

(i) Every closed invariant subspace contains at least one eigen-vector.

(ii) Every closed invariant subspace is also invariant under $T^{*}$.

(iii) If $\lambda_{1}, \lambda_{2}, \cdots$ are the distinct eigen-values of $T$, then $\sum_{i=1}^{\infty} \lambda_{1}^{n} \omega_{i}$ $=0, n \geqq 0$, and $\sum_{i=1}^{\infty}\left|\omega_{i}\right|<\infty$ imply $\omega_{1}=\omega_{2}=\cdots=0$.

Proof. We note that, since $T$ is normal, eigen-vectors belonging to different eigen-values are orthogonal to each other. Also, by hypothesis, the set of eigen-vectors is fundamental in the space. Hence,

Received by the editors July 13, 1951.

1 This paper is based on a portion of the author's dissertation, Harvard University, 1951. The author wishes to thank Professor G. W. Mackey for his guidance in the investigation. 
if $N_{i}$ denotes the subspace of $H$ consisting of the eigen-vectors which belong to $\lambda_{i}$, then $H=\sum_{i=1}^{\infty} N_{i}$, where $N_{i} \perp N_{j}$ if $i \neq j$. Further, if $T \phi=\lambda \phi$, then $T^{*} \phi=\bar{\lambda} \phi$.

Assume (i). Given a closed invariant subspace $C$, let $M$ be the closed subspace of $C$ generated by eigen-vectors. Suppose $M \not \equiv C$. Then $C^{\prime}=C \cap M^{\perp}$ is not empty. But if $x \in C^{\prime}$, then $T x \in C$, and if further $y \in M$, then $T^{*} y=k \cdot y$ for some scalar $k$, whence $(T x, y)$ $=\left(x, T^{*} y\right)=0$, and so $T x \in C^{\prime}$. Thus $C^{\prime}$ is invariant. It follows by hypothesis that $C^{\prime}$ contains an eigen-vector $\phi \neq 0$ and so $\phi \perp M$. But also $\phi \in M$. This contradiction shows that $M \equiv C$; hence (i) implies spectral synthesis. The converse obviously also holds.

Next, assume (ii). If $C$ is a closed invariant subspace not equal to $\{0\}$, then there exists an eigen-vector $\phi$ which is not orthogonal to $C$. We can write $\phi=\phi_{1}+\phi_{2}$, where $\phi_{1} \neq 0 \in C$, and $\phi_{2} \in C^{\perp}$. Hence $\lambda \phi_{1}$ $+\lambda \phi_{2}=\lambda \phi=T \phi=T \phi_{1}+T \phi_{2}$, and so $\lambda \phi_{2}-T \phi_{2}=T \phi_{1}-\lambda \phi_{1}$. Now $T \phi_{1}$ $\in C$ and, by (ii), $T \phi_{2} \in C^{\perp}$. Hence $T \phi_{1}-\lambda \phi_{1}=0$. Hence $T \phi_{1}=\lambda \phi_{1}$ and so $C$ contains at least one eigen-vector. By the preceding, then, spectral synthesis holds. The converse follows at once from the fact that every eigen-vector of $T$ is also an eigen-vector of $T^{*}$.

Finally, assume (iii). Let $C$ be a closed invariant subspace and let $x \in C$. We can write $x=\sum_{1}^{\infty} \chi_{i}$, where $\chi_{i} \in N_{i}$, the subspace of eigenvectors belonging to $\lambda_{i}$. Consider any $y$ now which is $\perp C$. We write $y=\sum_{1}^{\infty} \xi_{i}, \quad \xi_{i} \in N_{i}$. Since $T^{n} x \in C, n \geqq 0$, we have $\left(T^{n} x, y\right)$ $=\sum_{1}^{\infty} \lambda_{i}^{n}\left(\xi_{i}, \chi_{i}\right)=0$, or, setting $\left(\xi_{i}, \chi_{i}\right)=\omega_{i}, \sum_{1}^{\infty} \lambda_{i}^{n} \omega_{i}=0, n \geqq 0$. Since $\sum_{1}^{\infty}\left\|\chi_{i}\right\|^{2}<\infty, \sum_{1}^{\infty}\left\|\xi_{i}\right\|^{2}<\infty$, we further have $\sum_{1}^{\infty}\left|\omega_{i}\right|<\infty$. (iii) then implies that $\left(y, \chi_{i}\right)=\omega_{i}=0, i=1,2, \cdots$. It follows that each $\chi_{i} \in C$. Hence spectral synthesis holds.

The converse follows by reversing the argument, if we use the fact that to any sequence $\left\{\omega_{i}\right\}_{1}^{\infty}$ with $\sum_{1}^{\infty}\left|\omega_{i}\right|<\infty$, we can find sequences $\left\{\alpha_{i}\right\},\left\{\beta_{i}\right\}$ with $\sum_{1}^{\infty}\left|\alpha_{i}\right|^{2}, \sum_{1}^{\infty}\left|\beta_{i}\right|^{2}<\infty$, and $\alpha_{i} \beta_{i}=\omega_{i}$.

THEOREM 2. Given any infinite sequence of numbers $r_{i}$ with $0<r_{1}<r_{2}$ $<\cdots<r$, let $\Gamma_{i}$ be the circle $|z|=r_{i}$ in the complex plane. Then there exists a normal operator having its eigen-values on the circles $\Gamma_{i}$ such that spectral synthesis fails for this operator.

PROOF. By a construction due to Wolff [1], ${ }^{2}$ every function analytic in $|z|<1 / r_{2}$ and so in particular the function

$$
f(z)=-A_{1} /\left(z-\left(1 / r_{1}\right)\right)
$$

can be represented in the region $|z|<1 / 2 r$ by a Borel series $\sum_{2}^{\infty} A_{\nu} /\left(z-\alpha_{\nu}\right)$ where $\sum_{2}^{\infty}\left|A_{\nu}\right|<\infty$ and the $\alpha_{\nu}$ are distributed on

2 Numbers in brackets refer to the references cited at the end of the paper. 
the circles $|z|=1 / r_{i}$. Then

$0 \equiv \sum_{1}^{\infty} \frac{A_{\nu}}{z-\alpha_{\nu}}=-\sum_{m=0}^{\infty} z^{m} \sum_{\nu=1}^{\infty} \frac{A_{\nu}}{\alpha_{\nu}}\left(\frac{1}{\alpha_{\nu}}\right)^{m}, \quad \alpha_{1}=\frac{1}{r_{1}}$, for $|z|<\frac{1}{2 r}$ and so $\sum_{\nu=1}^{\infty} \lambda_{\nu}^{m} \omega_{\nu}=0, m=0,1,2, \cdots$, where $\omega_{\nu}=A_{\nu} / \alpha_{\nu}, \lambda_{\nu}=1 / \alpha_{\nu}$. Then $\sum_{\nu=1}^{\infty}\left|\omega_{\nu}\right|<\infty$ and each $\lambda_{\nu}$ lies on some circle $\Gamma_{i}$. (iii) now gives the assertion.

The last argument shows that the problem of spectral synthesis can be reduced to the unicity-problem for Borel series. As another application of this fact we have the following theorem.

THEOREM 3. Spectral synthesis holds if all the eigen-values $\lambda_{i}$ lie on a Jordan curve in the complex plane.

Proof. Suppose the contrary. Then there exists a sequence $\left\{\omega_{i}\right\}$ with $\sum_{1}^{\infty}\left|\omega_{i}\right|<\infty$, and $\sum_{1}^{\infty} \lambda_{i}^{n} \omega_{i}=0, n \geqq 0$. For large $|z|$, then, we have $0=\sum_{k=0}^{\infty} 1 / z^{k+1} \sum_{\nu=1}^{\infty} \lambda_{\nu}^{k} \omega_{\nu}=\sum_{1}^{\infty} \omega_{\nu} /\left(z-\lambda_{\nu}\right)$. On the other hand, it is known that a Borel series of this kind cannot vanish identically for large $|z|$ unless all its coefficients vanish [2]. The assertion follows by Theorem 1 .

THEOREM 4. If there exists no infinite subset $\lambda_{n_{i}}$ of the eigen-values $\lambda_{i}$ with $\left|\lambda_{n_{1}}\right|<\left|\lambda_{n_{2}}\right|<\cdots$, then spectral synthesis holds.

Proof. Suppose $\sum_{1}^{\infty} c_{i} \lambda_{i}^{n} \equiv 0, n \geqq 0, \sum_{i=1}^{\infty}\left|c_{i}\right|=A<\infty$. The set of numbers $\left|\lambda_{i}\right|$ with $c_{i} \neq 0$ is empty or has a maximum $r$, in consequence of the hypothesis, and there exists $r^{\prime}<r$ such that for any $\left|\lambda_{i}\right|<r$ we have $\left|\lambda_{i}\right|<r^{\prime}$. Let $\lambda_{r_{1}}, \lambda_{r_{2}}, \cdots$ be the sequence of eigen-values with modulus $r$. Then

$$
\sum_{i=1}^{\infty} c_{r_{i}} \lambda_{r_{i}}^{n}+\sum_{\left|\lambda_{j}\right|<r} c_{j} \lambda_{j}^{n}=0, \quad n \geqq 0 ; \quad \text { not all } c_{r_{i}}=0 .
$$

Therefore

$$
\left|\sum_{i=1}^{\infty} c_{r_{i}} \lambda_{r_{i}}^{n}\right| \leqq \sum_{\left|\lambda_{j}\right|<r}^{\infty}\left|c_{j}\right|\left|\lambda_{j}\right|^{n} \leqq A r^{\prime n}, \quad n \geqq 0 .
$$

Therefore

$$
\left|\sum_{i=1}^{\infty} d_{i} \xi_{i}^{n}\right| \leqq A\left(\frac{r^{\prime}}{r}\right)^{n}, \text { where } d_{i}=c_{r_{i}}, \xi_{i}=\frac{1}{r} \lambda_{r_{i}} .
$$

Then $\left|\xi_{i}\right|=1$, all $i$. We write $\xi_{j}=e^{2 \pi i \theta_{i}}$. By a theorem of Dirichlet [6] there exists for each $k, k=1,2, \cdots$, an integer $N_{k}>k$ such that $\left|N_{k} \theta_{j}-M_{j}^{k}\right|<1 / k, j=1,2, \cdots, k$, where the $M_{j}^{k}$ are integers. Given 
$l$, it follows that $\lim _{k=\infty} \xi_{i}^{n_{k}-l}=1$, all $i, n_{k} \rightarrow \infty$, where $n_{k}=N_{k}+l$. Therefore

$$
\sum_{1}^{\infty} d_{i} \xi_{i}^{l}=\lim _{k=\infty}\left(\sum_{i=1}^{\infty} d_{i} \xi_{i}^{l} \xi_{i}^{n_{k}-l}\right)=\lim _{k=\infty} \sum_{1}^{\infty} d_{i} \xi_{i}^{n_{k}}
$$

But $\left|\sum_{1}^{\infty} d_{i} \xi_{i}^{n_{k}}\right| \leqq \epsilon_{k}$ where $\epsilon_{k} \rightarrow 0$. Hence $\sum_{1}^{\infty} d_{i} \xi_{i}^{l}=0$. Also $\sum_{1}^{\infty}\left|d_{i}\right|$ $=\sum_{1}^{\infty}\left|c_{r_{i}}\right| \leqq A$, whence $d_{1}=d_{2}=\cdots=0$ by Theorem 3 , and so $c_{r_{i}} \equiv 0$; but this contradicts the choice of the $c_{r_{i}}$. Hence all $c_{i}=0$, and so spectral synthesis holds.

We next assert:

THEOREM 5. If there exists a sequence of polynomials $P_{n}$ with $P_{n}(T)$ converging weakly to $T^{*}$, then spectral synthesis holds for $T$. The converse statement is false.

Proof. Let $P_{n}(T)$ converge weakly to $T^{*}$. $\left(T^{n} x, y\right)=0, n \geqq 0$, implies $\left(P_{n}(T) x, y\right)=0$, whence $\left(T^{*} x, y\right)=0$ and so for every vector $x$, $T^{*} x$ lies in the smallest closed invariant subspace containing $x$. We next need the following lemma.

Lemma 1. If $\lambda_{1}, \lambda_{2}, \cdots$ are the eigen-values of $T$ and if $P_{n}$ is a sequence of polynomials, then weak and strong convergence of $P_{n}(T)$ to $T^{*}$ are both equivalent to

(a) $P_{n}\left(\lambda_{i}\right) \rightarrow \bar{\lambda}_{i}$, all $i$,

(b) $\left|P_{n}\left(\lambda_{i}\right)\right| \leqq K$, all $n$, $i$, for some $K$.

For if $\left(P_{n}(T) x, y\right) \rightarrow\left(T^{*} x, y\right)$ for all $x, y$, then $\sum_{i=1}^{\infty} P_{n}\left(\lambda_{i}\right)\left(\chi_{i}, \xi_{i}\right)$ $\rightarrow \sum_{i=1}^{\infty} \bar{\lambda}_{i}\left(\chi_{i}, \xi_{i}\right)$, for all $x=\sum_{1}^{\infty} \chi_{i}, y=\sum_{1}^{\infty} \xi_{i}$. Setting $B_{i}^{n}=P_{n}\left(\lambda_{i}\right)$ $-\bar{\lambda}_{i}$, we get

$$
\sum_{i=1}^{\infty} B_{i}^{n} \omega_{i} \rightarrow 0, \quad \text { all } \quad\left\{\omega_{i}\right\} \in l_{1} \quad \text { (i.e. with } \sum_{1}^{\infty}\left|\omega_{i}\right|<\infty \text { ). }
$$

For fixed $n,\left|B_{i}^{n}\right|<K_{n}$, all $i$, whence the $\left\{B_{i}^{n}\right\}_{i=1}^{\infty}$ are a pointwise converging sequence of bounded linear functionals on $l_{1}$, and so $\left|B_{i}^{n}\right|$ is bounded for all $i, n$. Thus (b) follows. Clearly also $\lim _{n=\infty} B_{i}^{n}=0$ for every $i$, and so (a) follows.

Conversely, assume (a) and (b). We have $\left\|\left(P_{n}(T)-T^{*}\right) x\right\|^{2}$ $=\sum_{1}^{\infty}\left|P_{n}\left(\lambda_{i}\right)-\bar{\lambda}_{i}\right|^{2}\left\|\chi_{i}\right\|^{2}$ which then approaches 0 as $n \rightarrow \infty$. This proves the lemma.

Let us now consider the case when the set of eigen-values consists of a dense set $\lambda_{i}$ on the unit circle and an infinite set $\sigma_{i}$ on the circle $|z|=1 / 2$. If $T$ is the corresponding operator, then spectral synthesis holds for $T$ by Theorem 4 . On the other hand, if $P_{n}(T)$ converges 
weakly to $T^{*}$, then by the lemma $P_{n}\left(\lambda_{i}\right) \rightarrow \bar{\lambda}_{i}, i=1,2, \cdots, P_{n}\left(\sigma_{i}\right)$ $\rightarrow \bar{\sigma}_{i}, i=1,2, \cdots$, and $\left|P_{n}\left(\lambda_{i}\right)\right|,\left|P_{n}\left(\sigma_{i}\right)\right|<K$ for some constant $K$, all $n, i$. Since the $\lambda_{i}$ are dense on $|z|=1,\left|P_{n}(z)\right| \leqq K,|z| \leqq 1$, all $n$. Hence some subsequence $P_{n_{k}}(z)$ converges uniformly in $|z|<3 / 4$ to $f(z)$ analytic in $|z|<3 / 4$. Since $P_{n}\left(\sigma_{i}\right) \rightarrow \bar{\sigma}_{i}$ and $\sigma_{i} \bar{\sigma}_{i}=1 / 4, f\left(\sigma_{i}\right)=\bar{\sigma}_{i}$ $=1 / 4 \sigma_{i}$, and so $f(z)-1 / 4 z$ has a set of zeros with a limit point on $|z|=1 / 2$. But then $f(z)$ provides an analytic continuation of $1 / 4 z$ to $|z|<3 / 4$, which is absurd. This proves Theorem 5 .

THEOREM 6. If a unitary operator $U$ has a fundamental set of eigenvectors, then there exist polynomials $P_{n}$ with $P_{n}(U)$ converging strongly to $U^{*}$.

Proof. As in the proof of Theorem 4, we construct a sequence of integers $N_{k}$ with $\lim _{k \rightarrow \infty} \lambda_{l}^{N_{k}}=1, i=1,2, \cdots$. Then $\lim _{k=\infty} \lambda_{i}^{N_{k}-1}$ $=\bar{\lambda}_{i}, i=1,2, \cdots$. Lemma 1 then yields our assertion, with $P_{n}(U)$ $=U^{N_{n}-1}$.

3. We shall say that a normal operator $T$ has "property $(\mathrm{P})$ " if every closed subspace invariant under $T$ is also invariant under $T^{*}$. We saw in Theorem 1 that if $T$ has a fundamental set of eigen-vectors, then spectral synthesis is equivalent to property (P). However, property $(\mathrm{P})$ has meaning for arbitrary normal operators and we now proceed to study this property in the general case.

By the spectral theorem for normal operators, we have for any $x, y \in H$ the formula $(T x, y)=\int \lambda \mu_{x y}(d \lambda)$ where $\mu_{x y}$ is a bounded completely additive set funcion defined for Borel sets in the plane and null outside the spectrum of $T$. Furthermore, to each Borel set $S$ is associated a projection-operator on $H, E(S)$, such that for all $x$ and $y$ we have $(E(S) x, y)=\mu_{x y}(S)$.

LEмма 2. $T$ has property (P) if and only if whenever we have $\int \lambda^{n} \mu_{x y}(d \lambda)=0$ for all $n \geqq 0$ for two vectors $x$ and $y$, we also have $\mu_{x y} \equiv 0$ as a measure.

Proof. Suppose property (P) holds and that for some $x, y$ we have $\int \lambda^{n} \mu_{x y}(d \lambda)=0, n \geqq 0$. Thus $\left(T^{n} x, y\right)=0, n \geqq 0$. Thus $y$ is orthogonal to the smallest closed invariant subspace containing $x$. This subspace now also contains $T^{* n} x, n \geqq 0$, and so $0=\left(T^{* n} x, y\right)=\int \bar{\lambda}^{n} \mu_{x y}(d \lambda)$, $n \geqq 1$. It follows that $0=\int f(\lambda) \mu_{x y}(d \lambda)$ for every continuous function $f(\lambda)$ and so $\mu_{x y} \equiv 0$.

Conversely, if property $(\mathrm{P})$ fails, then there exists a closed invariant subspace $C$ and some $x \in C$ with $T^{*} x \notin C$. Hence for some $y$ we have $y \perp C$ and not $y \perp T^{*} x$. This means that $\int \lambda^{n} \mu_{x y}(d \lambda)=0, n \geqq 0$, while $\int \bar{\lambda} \mu_{x y}(d \lambda) \neq 0$ and so $\mu_{x y} \not \equiv 0$. This proves the lemma. 
We note that the condition of this lemma specializes down to condition (iii) of Theorem 1 if $T$ has a fundamental set of eigenvectors.

It is impossible to characterize those operators for which property (P) holds in terms of their spectrum alone, since two operators may have the same spectrum while $(\mathrm{P})$ holds for one and fails for the other. However, we have the following sufficient condition:

THEOREM 7. If the spectrum of $T$ does not separate the plane and has no interior, then (P) holds.

Proof. Let $P$ be a polynomial. Then $\left\|P(T)-T^{*}\right\|=$ the least upper bound of $|P(z)-\bar{z}|$ taken over all $z$ in the spectrum of $T$. It is known that every function continuous on a compact set without interior which does not separate the plane can be approximated uniformly on this set by polynomials in $z[3]$. Since $\bar{z}$ is a continuous function, our hypothesis yields that $T^{*}$ is a limit in norm of polynomials in $T$. It clearly follows from this that $(P)$ holds.

We now introduce the following definition and notation: Let $m$ be a set-function defined for Borel sets of the plane and let $T$ be a normal operator. For any set $S$, let $E(S)$ be the projection associated to $S$ by the spectral representation for $T$.

Definition. $m$ is called "absolutely continuous with respect to the spectral measure of $T$ ", and we write $m>T$ if, whenever $E(S)=0$ for a certain Borel set $S$, we also have $m(S)=0$.

We shall make use of the following known result about normal operators: Let $T$ be a normal operator and $m$ a non-negative measure with $m>T$. Then for any bounded Borel measurable complex function $f(\lambda)$ there exist vectors $x, y$ such that for any set $S, \mu_{x y}(S)$ $=\int_{S} f(\lambda) m(d \lambda)$. If $f(\lambda) \equiv 1, y=x$ and so $m(S)=\mu_{x x}(S)$.

THEOREM 8. Let $d$ be a circular disk contained in the spectrum of $T$ and let $m$ be the measure which assigns to each set $S$ the Lebesgue measure of the intersection of $S$ and $d$. If $m>T$, then (P) fails for $T$.

Proof. Let $d$ be the set of $z$ with $\left|z-z_{0}\right| \leqq r$. Set $f(z)=z-z_{0}$ for $z \in d$ and $f(z)=0$ elsewhere. By the preceding, there exist vectors $u$ and $v$ with $\mu_{u v}(d z)=f(z) m(d z)$ and so

$$
\int z^{n} \mu_{u v}(d z)=\int z^{n} f(z) m(d z)=\iint_{\left|z-z_{0}\right| \leqq r} z^{n}\left(z-z_{0}\right) d x d y .
$$

But $\iint_{\left|z-z_{0}\right| \leqq r} z^{n}\left(z-z_{0}\right) d x d y=0, n \geqq 0$, while $\mu_{u v} \not \equiv 0$. Thus (P) fails.

Leмma 3. A unitary operator $U$ has property $(\mathrm{P})$ if and only if there 
does not exist an orthonormal sequence $\left\{\phi_{k}\right\}_{-\infty}^{\infty}$ with $U \phi_{k}=\phi_{k+1}$ for all $k$.

Proof. Suppose there exists such a set $\left\{\phi_{k}\right\}$. Then, if $C$ is the subspace spanned by $\phi_{k_{0}}, \phi_{k_{0}+1}, \phi_{k_{0}+2}, \cdots, C$ is invariant under $U$ while $U^{-1} \phi_{k_{0}}=\phi_{k_{0}-1} \notin C$, and so $C$ is not invariant under $U^{-1}=U^{*}$, whence $U$ lacks property $(\mathrm{P})$. Conversely, assume there exists an invariant subspace $C$ with $\phi \in C, U^{*} \phi=U^{-1} \phi \notin C$. Then $U^{-1} \phi=\psi+\phi_{1}$, $\psi \in C, \phi_{1} \neq 0, \perp C$.

Now $U^{n} \phi_{1}=U^{n-1} \phi-U^{n} \psi \in C, n \geqq 1$, and so $\phi_{1} \perp U^{n} \phi_{1}, n \geqq 1$. Hence if $\phi_{k}=U^{k} \phi_{1}, k=0, \pm 1, \pm 2, \cdots,\left(\phi_{k}, \phi_{l}\right)=\left(U^{k} \phi_{1}, U^{l} \phi_{1}\right)=\left(\phi_{1}, U^{l-k} \phi_{1}\right)$ $=0, l \neq k$, or the $\phi_{k}$ form an orthonormal set and $U \phi_{k}=\phi_{k+1}$.

Theorem 9. Let $T$ have its spectrum on a closed rectifiable curve $\Gamma$ which bounds a simply-connected region $D$. Let $m$ be the unique measure on the plane which is null outside of $\Gamma$ and which assigns to each arc of $\Gamma$ its length. (P) then fails if and only if $m>T$.

Proof. Let $\phi$ map $D$ conformally on $|z|<1$. Then $\phi$ maps $D+\Gamma$ homeomorphically on $|z| \leqq 1$, and a set $S$ on $\Gamma$ has $m(S)=0$ if and only if the linear measure of $S^{\prime}$ is $0, S^{\prime}$ being the image set of $S$ on $|z|=1[4]$.

Let us define an operator $U$ by $(U x, y)=\int_{\Gamma} \phi(\lambda) \mu_{x y}(d \lambda)$ where $\mu_{x y}$ comes from the spectral representation of $T$. Since $|\phi(\lambda)|=1$ if $\lambda \in \Gamma$, $U$ is unitary and so we can write $(U x, y)=\int \xi \mu_{x y}^{\prime}(d \xi)$, where $\mu_{x y}^{\prime}$ is concentrated on the unit circle. It follows that for any $F(\xi)$ continuous on $|\xi|=1, \int_{\Gamma} F(\phi(\lambda)) \mu_{x y}(d \lambda)=\int_{|\xi|=1} F(\xi) \mu_{x y}^{\prime}(d \xi)$. Hence if $S$ is any set on $\Gamma$ and $S^{\prime}=\phi(S)$, then $\mu_{x y}(S)=\mu_{x y}^{\prime}\left(S^{\prime}\right)$; in particular for a given $S, E(S)=0$ if and only if $E^{\prime}\left(S^{\prime}\right)=0$.

(1) (P) fails for $T$ if and only if (P) fails for $U$. For let (P) fail for $T$. Then there exist $x, y$ with $\left(T^{n} x, y\right)=\int_{\Gamma} \lambda^{n} \mu_{x y}(d \lambda)=0, n \geqq 0$, and $\mu_{x y} \not \equiv 0$. Therefore $\mu_{x y}^{\prime} \not \equiv 0$ and $\left(U^{m} x, y\right)=\int_{\Gamma} \phi^{m}(\lambda) \mu_{x y}(d \lambda)=0, m \geqq 0$, since $\phi^{m}$ is analytic in $D$ and continuous in $D+\Gamma$ and so, by a theorem of J. L. Walsh [5], can be approximated uniformly on $\Gamma$ by a linear combination of powers of $\lambda$. Thus (P) fails for $U$. A dual argument shows the converse.

(2) (P) fails for $U$ if and only if linear measure $s$ on the circle is absolutely continuous with respect to the spectral measure of $U$. For if (P) fails for $U$, Lemma 3 yields the existence of $x \neq 0$ with $\left(U^{k} x, x\right)$ $=0, k \neq 0$. Then $\int_{|\xi|=1} \xi^{k} \mu_{x x}^{\prime}(d \xi)=0, k \neq 0$, and hence $\mu_{x x}^{\prime}$ equals linear measure on the circle up to a positive constant, and so $s>U$.

Conversely, let $s>U$. Then, as we noted above, there exists $x$ with $s\left(S^{\prime}\right)=\mu_{x x}^{\prime}\left(S^{\prime}\right)$, for all $S^{\prime}$ on $|\xi|=1$. Hence 


$$
\left(U^{k} x, x\right)=\int_{|\xi|=1} \xi^{k} \mu_{x x}^{\prime}(d \xi)=\int_{|\xi|=1} \xi^{k} d s=\int_{0}^{2 \pi} e^{i k \theta} d \theta=0, \quad k \neq 0,
$$

and so (P) fails, by Lemma 3.

(3) $s>U$ if and only if $m>T$. For let $s>U$. If now $E(S)=0$ for some $S$ on $\Gamma$, then $E^{\prime}\left(S^{\prime}\right)=0$, where $S^{\prime}=\phi(S)$. Hence $s\left(S^{\prime}\right)=0$, by assumption. As we observed above, this implies that $m(S)=0$. Thus $m>T$. The converse follows similarly.

(1), (2), and (3) together now give the assertion.

A. Beurling [7] has discussed a problem related to the one we have been considering. He concerned himself in particular with a bounded operator on Hilbert space which has a fundamental set of eigen-vectors and the set of whose eigen-values fills the open unit circle.

\section{REFERENCES}

1. T. Wolff, C. R. Acad. Sci. Paris vol. 173, pp. 1327-1328.

2. T. Hall, Thesis, Uppsala, 1950.

3. M. Lavrentieff, Sur les fonctions d'une variable complexe représentable par des séries de polynoms, Actualités Scientifiques et Industrielles, no. 441, 1936.

4. F. and M. Riesz, Über Randwerte einer analytischen Funktion, $4^{\circ}$ Congrès des Math. Scand., 1916, pp. 27-44.

5. J. L. Walsh, Interpolation and approximation by rational functions in the complex domain, Amer. Math. Soc. Colloquium Publications, vol. 20, p. 36.

6. E. C. Titchmarsh, The theory of functions, pp. 295-296.

7. A. Beurling, On two problems concerning linear transformations in Hilbert space, Acta Math. vol. 81.

YALE UNIVERSITY 